Article

\title{
The Design and Application of Non-Pressure Infiltrating Irrigation in Desertification Control
}

\author{
Yongping $\mathrm{Qu}{ }^{1, *}$ and Zengzhi Zhang ${ }^{2}$ \\ 1 School of Materials Science and Engineering, North University of China, Taiyuan 030051, China \\ 2 Research Institute of Ecological and Functional Materials, China University of Mining and \\ Technology (Beijing), Beijing 10083, China; z.zengzhi@163.com \\ * Correspondence: quyongping1989@163.com
}

Received: 14 December 2019; Accepted: 15 February 2020; Published: 19 February 2020

check for updates

\begin{abstract}
The key restriction factor of desertification is water control. Non-pressure infiltrating irrigation was invented aimed at the efficient use of water, and its core technology is the key water-conducting material prepared by montmorillonite and polyacrylamide. In this study, both the water absorption and desorption performances of the key material were tested, and then the binding condition of the water-conducting materials and the fiber substrate was also studied. Lastly, the water infiltrating performances and its application in deserts were tested. Non-pressure infiltrating irrigation could self-regulate the water conducting speed according to the external soil deserticola, but also significantly reduce water consumption. The results provide a reference for the application of this new water-saving irrigation technology, which could play an important role in desertification control.
\end{abstract}

Keywords: desertification; sand fixation; psammophytes; irrigation; soil moisture; self-dynamic regulation

\section{Introduction}

Desertification is one of the most threatening problems for humans in the 21st century [1]. The global desertification area has reached 36 million square kilometers and keeps increasing by $1.1 \%$ every years [2]. At present, the commonly used sand fixation technologies mainly include engineering sand fixation, chemical sand fixation and biological sand fixation [3]. By laying "grass grids", engineering sand fixation could establish sand barriers to block the forward movement of wind-driven sand with low cost. However, its protection height is limited and it is easy to be buried by quicksand, so it can only be used as a temporary auxiliary technology in highway and railway construction [4]. By spraying sand-fixing agents, chemical sand fixation can consolidate the sand layer and it has quite good sand fixation effect [5]. However, its durability is poor because of the use of polymer materials, which age easily. Among all the existing measures, the most thoroughgoing method is biological sand fixation, which is mainly based on the planting of sand-fixing seedlings to fix the loose sand [6]. However, it is limited in the practical promotion because of its high cost. In deserts, the natural precipitation is not enough for plant demands, so artificial irrigation is essential, resulting in the high cost of biological sand fixation [7]. So, it is necessary to develop water saving irrigation techniques, such as drip irrigation and infiltrating irrigation [8-11].

For drip irrigation and infiltrating irrigation, the average water output speed could respectively

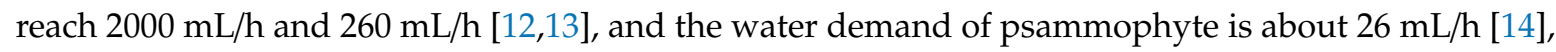
which is much lower than the water outputs of irrigation systems. Furthermore, the water output is passive and relies on pressure, so it is hard to realize water supply according to the plant demands [15]. For drip irrigation and most infiltrating irrigation methods, the trace water outputs are realized by mechanical micro channels [16]. In order to achieve the highest water use efficiency, the most objective 
irrigation method is an automatic water supply, which means adjusting the water output speed according to plant demands.

Herein, we invented a new kind of water-saving irrigation technology—non-pressure infiltrating irrigation. Traditional irrigation methods mainly conduct water by the synergistic effects of impressed pressure and mechanical micro channels. Non-pressure infiltrating irrigation mainly relies on the complex interactions of the composite material and water. Thus, the technology could realize the automatic and scientific regulation of water conduction according to water demands. This paper mainly focused on the design of the "non-pressure infiltrating irrigation" system and on the self-regulating water conductivity of the irrigation system. Furthermore, its application in a desert was also studied.

\section{Materials and Methods}

\subsection{Materials}

Sodium-based montmorillonoid (MMT) (200 mesh, produced by Zhejiang Fenghong New Materials Co., Ltd.), polyacrylamide (PAM) (Analytical Reagent, produced by SNF Co., Ltd.), and cotton fibers (Chemically Pure, produced by Push Biotechnology Co., Ltd.) were used to prepare the water-conducting fibers.

\subsection{The Design of Infiltrating Irrigation System}

The key water-conducting material was composed of PAM and MMT. The preparation process is shown in Figure 1. First of all, $0.2 \%$ PAM solution was prepared by stirring and heating at $40{ }^{\circ} \mathrm{C}$ for $20 \mathrm{~min}$, and then left for $12 \mathrm{~h}$. Then 1\% MMT suspension was prepared by magnetically stirring for $20 \mathrm{~min}$ and ultrasonically stirring for $10 \mathrm{~min}$. After that, the PAM solution was slowly poured into MMT suspension by stirring for $15 \mathrm{~min}$ until the mixture was uniform. The optimum ratio of PAM to MMT was 1:4. Secondly, the cotton fibers were soaked in $14 \%$ sodium hydroxide solution for $30 \mathrm{~min}$ to remove the oil adsorbed on the surface, then neutralized in $5 \%$ glacial acetic acid and washed with clean water. Then the pretreated cotton fibers were soaked in PAM/MMT mixed colloid solution for $10 \mathrm{~min}$ and then dried in an oven for $8 \mathrm{~h}$. Lastly, the water-conducting fibers were filled into the self-made infiltrating irrigation emitter, and the infiltrating irrigation system was composed of the emitters and the pipelines. The structure diagram of infiltrating irrigation emitter was shown in Figure 2, and the diagrammatic sketch is shown in Figure 3.

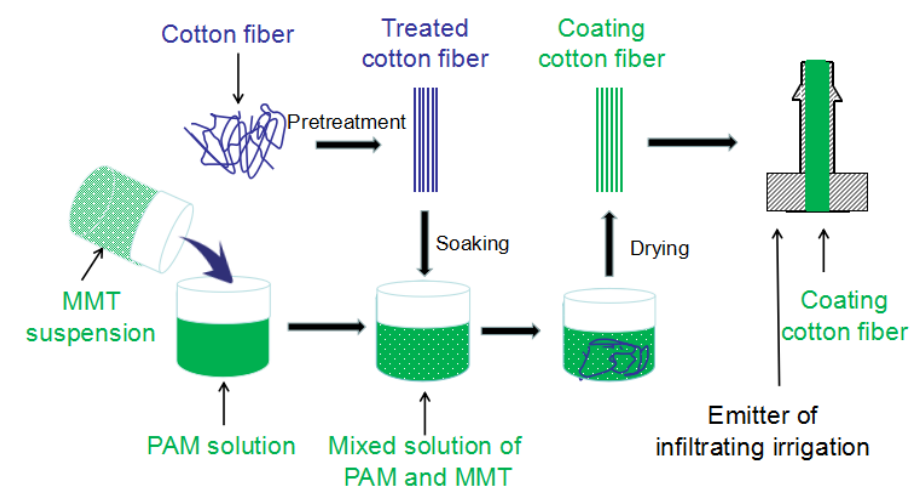

Figure 1. The preparation process of the water-conducting material. 

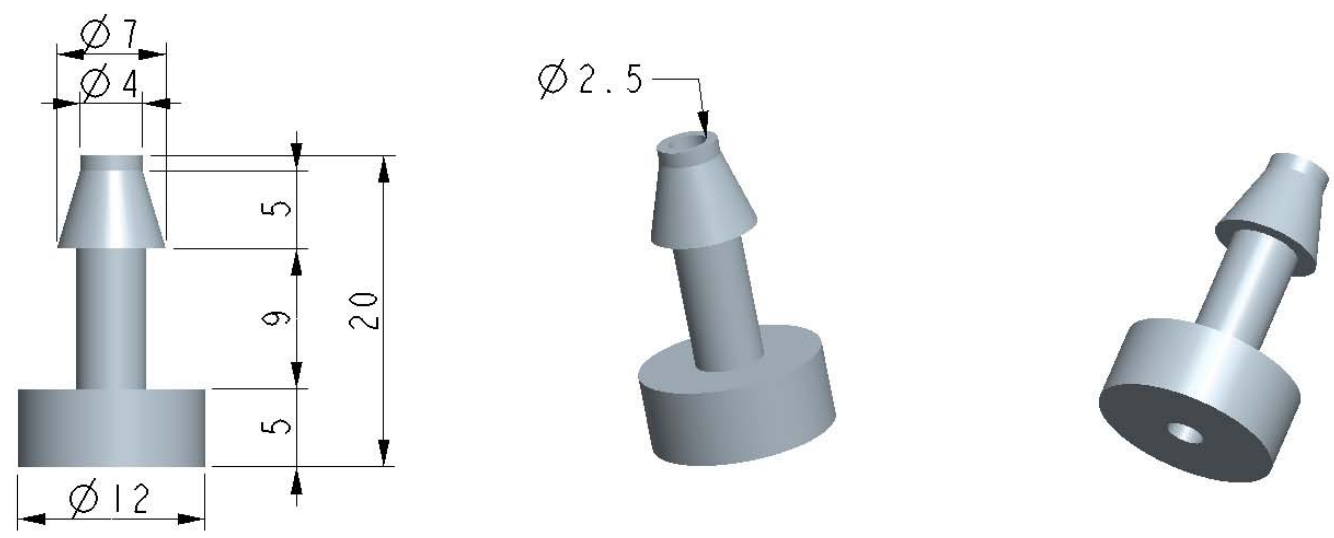

Figure 2. The three-dimensional structure diagram of infiltrating irrigation emitter (Unit: mm).

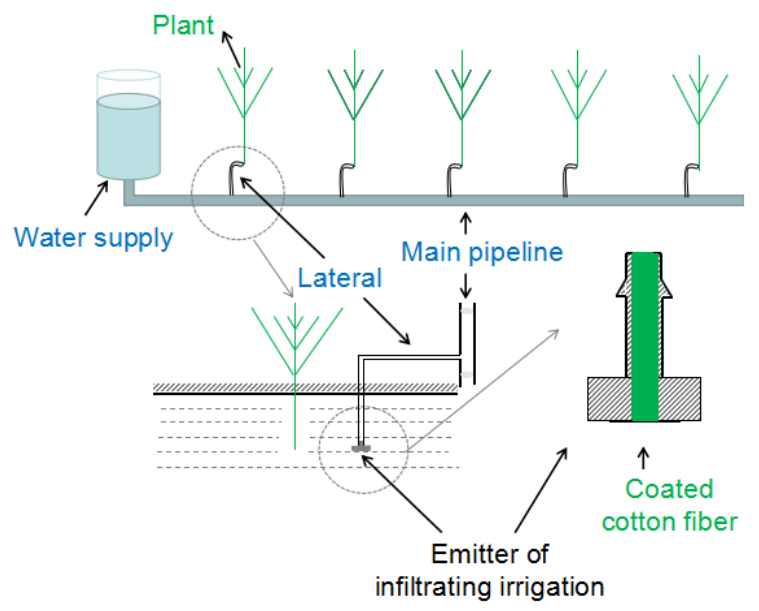

Figure 3. The diagrammatic sketch of the infiltrating irrigation system.

\subsection{The Water Absorption and Desorption Tests}

Water absorption tests: MMT/PAM slurry was dried and formed into a solid block of $2 * 2 * 0.5 \mathrm{~cm}^{3}$. Afterwards the block was dried to constant weight at $60{ }^{\circ} \mathrm{C}$, and then wrapped with gauze and soaked in water. The block mass (Mt) was measured and recorded regularly until it was absorbed to a constant weight.

Water desorption tests: The samples were divided into two groups after fully absorbing water. Then two groups were respectively put into air and dry sand to lose water, and their masses $(\mathrm{Mg}$ and Ms) were measured and recorded regularly.

The sample mass before absorbing water was named $M_{0}$, and the mass with saturation water absorption was named $M_{1}$. Then the calculating formulas of water absorption capacity ( $\left.Q \mathrm{t}\right)$, water loss capacity in air $(Q g)$ and water loss capacity in sand $(Q s)$ were as follows:

$$
\begin{aligned}
& Q_{\mathrm{t}}=\frac{M_{t}-M_{0}}{M_{0}}, \\
& Q_{\mathrm{g}}=\frac{M_{1}-M_{\mathrm{g}}}{M_{0}}, \\
& Q_{\mathrm{s}}=\frac{M_{1}-M_{s}}{M_{0}},
\end{aligned}
$$




\subsection{The Water Infiltrating Tests}

We studied the binding condition of the materials and the fibers by optical microscope and scanning electron microscope (SEM). Then, we prepared the emitters to test the water infiltrating performance. The tests were taken in a self-made sandbox. The emitters were buried into sand to a depth of $10 \mathrm{~cm}$ and no external pressure was applied at the water source. Five parallel samples were designed and the water outputs were measured every two hours.

In the dry-wet alternating test, the emitters were first buried into dry sand, and when water output trended to be stable after three days, the emitters were buried into dry sand again. This was repeated three times to test the self-regulating water conductivity performances under the dry-wet alternating environment. The water outputs were tested daily.

\subsection{The Application Tests in Desert Conditions}

Desert field tests: Tests were conducted in Ulanbuh desert, Dengkou County, Inner Mongolia in 2016. The basic climatic conditions of the test area are shown in Table 1. Cistanche deserticola was chosen as the experimental subject because of its extremely rigorous water demands. It is a kind of parasitic plant that invades the roots of Sacsaoul, which is the most common sand-fixing plant. According to our previous experiments, the optimum soil moisture content is $15 \%$ 20\% for Cistanche deserticola at the inoculation period and growth period, while slightly more or less water reduces the Cistanche deserticola survival rates. Based on the previous experimental experience, the inoculation depth was set to $50 \mathrm{~cm}$. The soil water contents of upper sand layers are not steady and easily influenced, which are easy to be influenced by solar irradiation. Three experiment groups were set for comparison with 100 plants in every group. Water storage pit irrigation and drip irrigation were respectively used in Group A and B, while non-pressure infiltrating irrigation was used in Group C. In Group A and B, we chose to irrigate when the soil water content at a depth of $50 \mathrm{~cm}$ dropped below $15 \%$ for a week. To a certain extent, the irrigation time and frequency were associated with the weather conditions. After precipitation, the soil water contents of the sand layers below $50 \mathrm{~cm}$ were also relatively high, so no irrigation would be chosen after precipitation. When there was no precipitation, we would monitor soil moisture at $50 \mathrm{~cm}$ underground every day to determine the irrigation timing. Therefore, the frequency of irrigation was related to the weather. The total irrigation number for Group A was 4 during the inoculation period of 6 months. According to our previous experiments, the inoculation "seed package" of Cistanche deserticola was generally placed $50 \mathrm{~cm}$ deep near the roots of Sacsaoul, the emitter for infiltrating irrigation was also placed $50 \mathrm{~cm}$ underground. In order to reduce the effect of water storage change on the water pressure, the bucket was marked so that when the water level dropped to $15 \mathrm{~cm}$ water was added to take the water level up to $20 \mathrm{~cm}$, and water complement was conducted when the liquid level dropped to $15 \mathrm{~cm}$. Therefore, the liquid level was kept within a small interval of 15 to $20 \mathrm{~cm}$ high. At last, the survival rates were counted regularly.

Table 1. Climatic conditions of the test site.

\begin{tabular}{cc}
\hline Ulanbuh Desert & Statistic \\
\hline Maximum annual precipitation & $150.3 \mathrm{~mm}$ \\
Minimum annual precipitation & $33.3 \mathrm{~mm}$ \\
Average annual precipitation & $102.9 \mathrm{~mm}$ \\
Average annual evaporation & $2258.8 \mathrm{~mm}$ \\
Maximum temperature & $39{ }^{\circ} \mathrm{C}$ \\
Minimum temperature & $-29.6^{\circ} \mathrm{C}$ \\
Average temperature & $7.8^{\circ} \mathrm{C}$ \\
\hline
\end{tabular}




\section{Results and Discussion}

\subsection{Water Absorption and Desorption Performances of the Water-Conducting Material}

As seen in Figure 4, the material absorbed water rapidly from the beginning to the 40th minute when the water absorption speed began to decrease, and then gradually becoming saturated after $130 \mathrm{~min}$. After that, the water absorption rate had no obvious change with time, the saturated water absorption capacity reached $36 \mathrm{~g} / \mathrm{g}$. Figure 5 shows the water desorption performances in air and in dry sand. The material maintained a high speed of water lose from the beginning to the 70th minute, and then the speed of water loss gradually decreased until the water loss process basically finished at 170th minute. As the suction in dry sand is greater than in air, the maximum water loss reached $25 \mathrm{~g} / \mathrm{g}$ in air and $27 \mathrm{~g} / \mathrm{g}$ in sand. The most important quality for the key materials is the water-conducting performance, so its water absorption and water desorption performances should be balanced. For the water-conducting material, both water absorption performance and water desorption performance were acceptable.

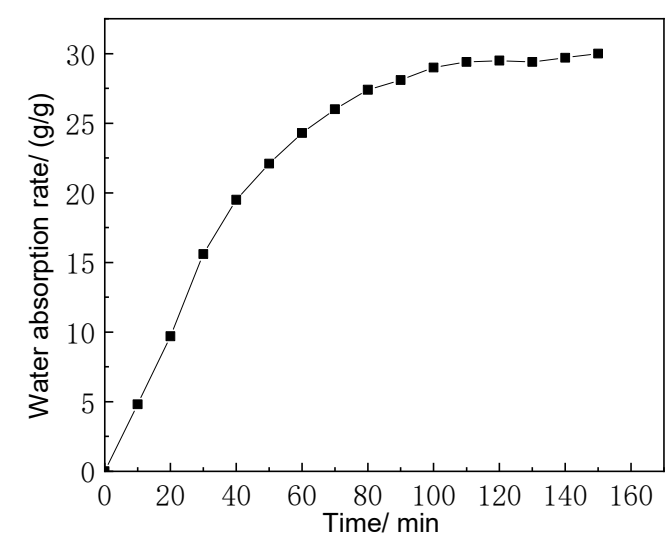

Figure 4. The water absorption property.

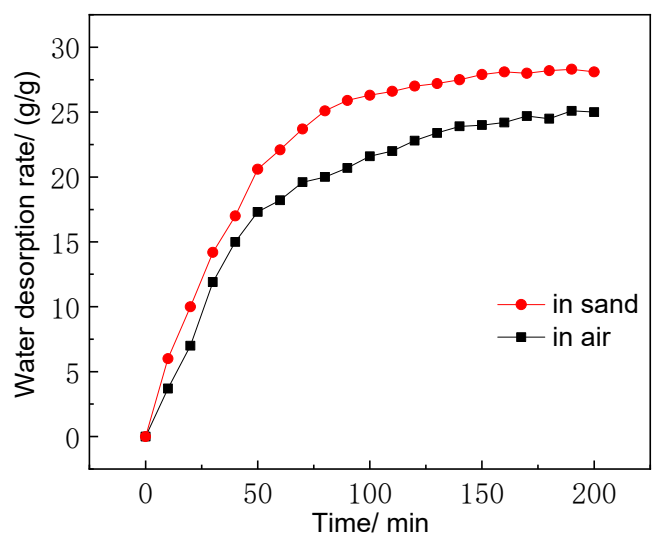

Figure 5. The water desorption property in air and in sand.

\subsection{The Coating Performances of the Water-Conducting Materials on the Cotton Fibers}

As a key part of the irrigation emitters, the coating performance of the materials on the cotton fibers is very important. In order to conduct water efficiently, the materials should be evenly coated on the fiber surface. As shown in Figure 6a, the cotton fibers were distributed randomly, and their surfaces were smooth. Figure $6 \mathrm{~b}$ shows the fibers after the coating process. Through the connection of the water-conducting materials, the fibers had formed a bundle of fiber aggregate after coating. Figure $6 c$,d show the SEM images of coated fibers. In this figure, the state of the coating and the fiber body could be seen more clearly. Almost all the fibers have been coated. Almost all the fiber substrates 
were uniformly coated with the water-conducting materials, and the materials arranged compactly to form the water conducting channel.

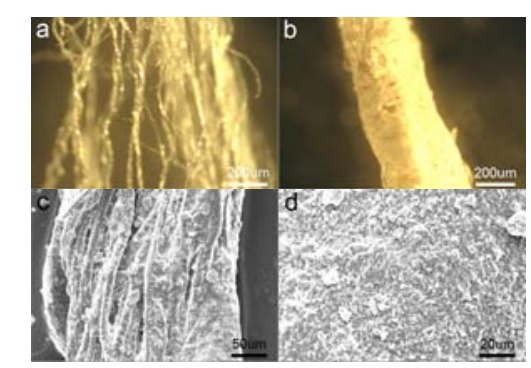

Figure 6. Optical microscope photographs of the cotton fibers and the coated fibers (a,b). The SEM photographs of the coated fibers (longitudinal section c) (transverse section $\mathbf{d}$ ).

\subsection{Water Infiltration Performance of the Irrigation System}

As Figure 7 shows, the average water output of the emitters showed a sharp change within $24 \mathrm{~h}$. A maximum of $80 \mathrm{~mL} / \mathrm{h}$ was achieved in the first two hours. Then the water output speed decreased rapidly until the 10th hour with a water output of $27 \mathrm{~mL} / 2 \mathrm{~h}$. After that, the decline slowed down and the water output rate remained about $20 \mathrm{~mL} / 2 \mathrm{~h}$, until it declined to a minimum of $15 \mathrm{~mL} / 2 \mathrm{~h}$ at the 24th hour. Figure 8 shows the changes of water outputs over a week. In the first day, the water output was $386 \mathrm{~mL}$, then it declined and remained around $160 \mathrm{~mL} / \mathrm{d}$ from the second day. According to Figures 7 and 8 , the emitters could self-reduce the water output speed when the external soil moisture content increased.

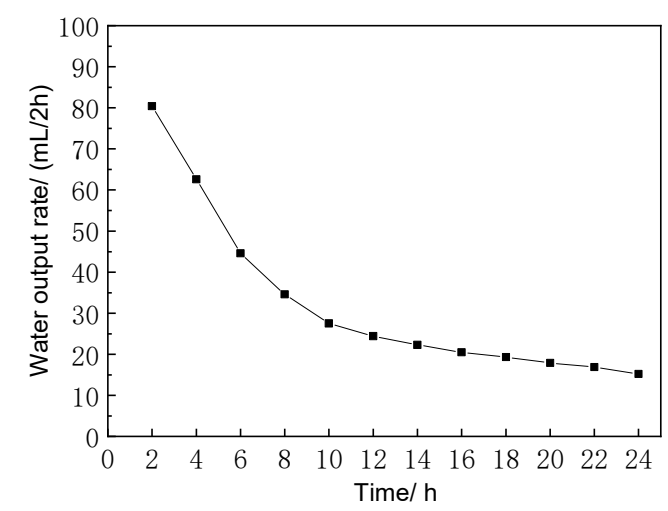

Figure 7. The average water output per emitter over $24 \mathrm{~h}$.

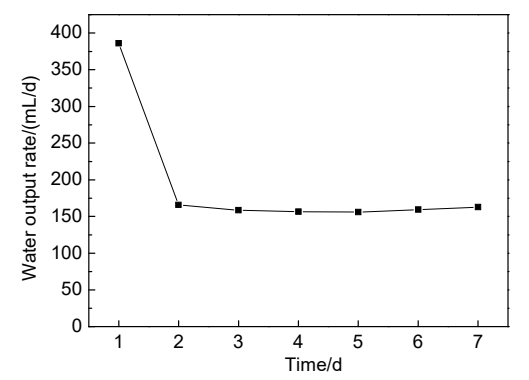

Figure 8. The average water output per emitter over a week.

Figure 9 shows the three dry-wet cycles of the emitters. As shown, the water output declined from $384 \mathrm{~mL} / \mathrm{d}$ to $155 \mathrm{~mL} / \mathrm{d}$ and tended to be stable in the first cycle, which indicated that the emitters could self-reduce the water output speed when soil moisture content increased. When the soil moisture content decreased in the second cycle, the water output recovered immediately to $388 \mathrm{~mL} / \mathrm{d}$, which 
meant that the emitters could self-hasten the water output speed when soil moisture content decreased. Overall, the three cycles showed that the wet-dry alternating performances of the emitters were good, which indicated that the non-pressure infiltrating irrigation system could indeed self-regulate the water-conducting rate according to the external soil moisture.

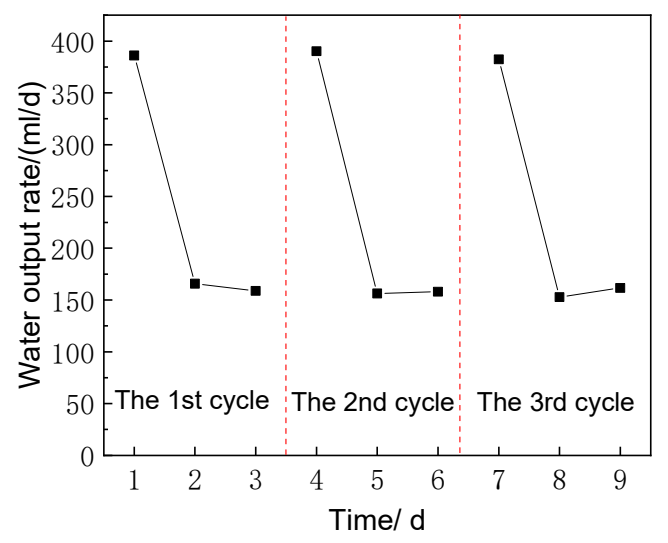

Figure 9. The average water outputs of emitters with 3 dry-wet cycles.

\subsection{The Application Performances in Deserts}

On the basis of the above experiments, the non-pressure infiltrating irrigation system was applied to the planting of Cistanche deserticola in deserts. Figure 10 shows some photos in desert tests. As seen in Figure 10a, the water source was supplied by two $40 \mathrm{~L}$ buckets, which could serve for $0.2 \mathrm{~km}^{2}$. There was no need to apply external pressure at the water resource, buckets were placed slightly above the pipeline, so that the water could flow freely in the pipe. Figure 10b,c show the layout of the pipeline, the main pipeline was arranged neatly and each plant was supplied with a branch tube to conduct water. As seen in Figure 10d, the capillary tube extended underground and the emitter was placed near the roots of the plant to reduce water waste. Figure 10e,f shows the successfully inoculated Cistanche deserticola. The white lumps were Cistanche deserticolas, and they were parasitic on the roots of Sacsaoul. As seen in Figure 10g, the mature Cistanche deserticolas were collected for statistical measurement. Figure 10h shows the flowers of Cistanche deserticola, and the pillars below the Sacsaoul plants were the flowering Cistanche deserticolas. The successful inoculated Cistanche deserticola would continue to grow out of the ground until it stopped growing after flowering.

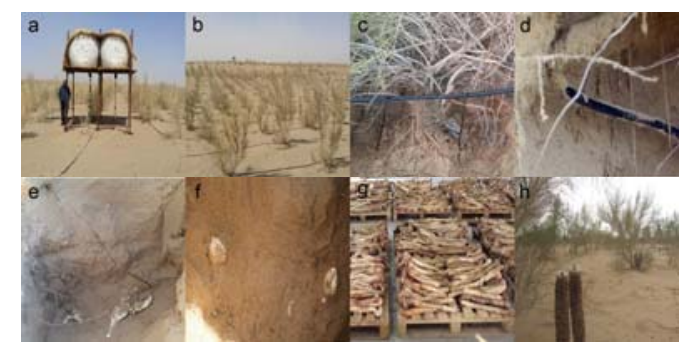

Figure 10. The photos in deserts: (a-d) the design of non-pressure infiltrating irrigation system; (e) the Cistanche deserticola parasitic on the roots of Sacsaoul; (f) the successfully inoculated Cistanche deserticola; (g) the Cistanche deserticola harvest; (h) the Cistanche deserticola flowering.

Figure 11 shows the water consumptions and the survival rates of Cistanche deserticola with different irrigation methods. Group A and Group B were respectively the water storage pit irrigation and drip irrigation, and Group C was non-pressure infiltrating irrigation. So, Group A and B were with intermittent water supply, while Group $C$ was with continuous water supply. As shown, water consumption and survival rate of Group A were $1600 \mathrm{~L}$ and $18 \%$. For group B, survival rate increased to $25 \%$, and water consumption had a $50 \%$ increase to $2400 \mathrm{~L}$. Group C had the highest survival 
rate of $83 \%$, much higher than group A and group B, while its water consumption was the lowest at $105 \mathrm{~L}$. Therefore, non-pressure infiltrating irrigation could greatly improve the survival rate, and at the same time it could also significantly reduce water consumption. Figure 12 shows the average grass heights of Cistanche deserticola with different irrigation methods. The grass heights of all the three groups were about $70 \mathrm{~cm}$, which meant different irrigation methods had little effect on grass heights of Cistanche deserticola.

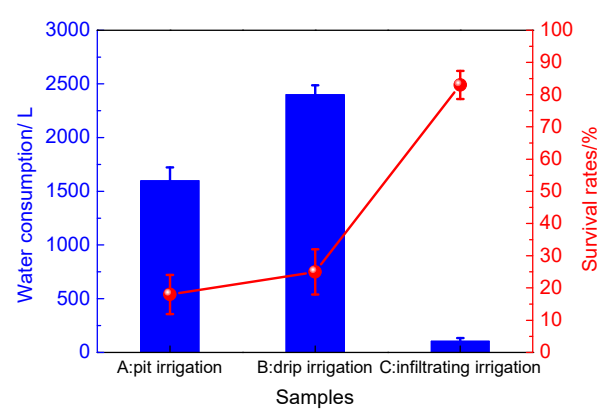

Figure 11. The survival rates and water consumptions of different groups.

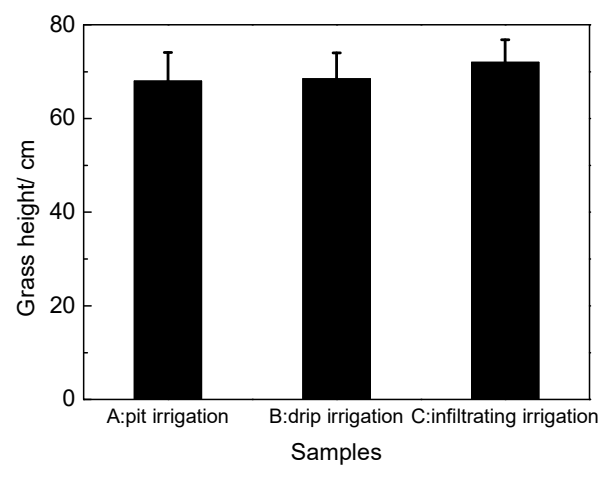

Figure 12. The average grass heights of different groups.

Compared with the traditional infiltrating irrigation techniques, the cost of non-pressure infiltrating irrigation increases very slightly, which is caused by the emitter. The water-conducting material in the emitter is prepared by PAM and MMT at the ratio of 1:4. The cost of PAM is $\$ 2000$ per tonne, and MMT is \$200 per tonne. So, after cost accounting, the cost increase of each emitter is no more than $20 \%$ compared with the traditional emitters. Moreover, the cost of emitter accounts for only $3 \%$ of the cost of the whole irrigation system. So, the cost increase of the whole irrigation system is almost negligible. The cost of non-pressure infiltrating irrigation is about $\$ 2223$ per hectare, which is close to the existing infiltrating irrigation technologies. The cost is too high for the application in desertification control, so infiltrating irrigation is always used for the cultivation of high value-added plants such as fruits and vegetables. Non-pressure infiltrating irrigation should be mainly used for the cultivation of the precious Chinese herbal medicines, which only grow in deserts, such as Cistanche deserticola. Cistanche deserticola is parasitic plant, which needs to parasitize the roots of Sacsaoul, the latter which is a typical sand-fixing species. Therefore, in order to obtain Cistanche deserticola, it is necessary to plant Sacsaoul first, which promotes the planting of sand-fixing seedlings. By this way, we achieve the purpose of desertification control.

\section{Conclusions}

We are certain that the materials could self-regulate the water conducting speed according to the external soil moisture, so the non-pressure infiltrating irrigation system could keep soil moisture within a constant range. The experiment in the desert showed that the survival rates of Cistanche deserticola could be increased to more than $80 \%$ by using a non-pressure infiltrating irrigation system, 
which was much higher than $18 \%$ by pit irrigation and $25 \%$ by drip irrigation. Moreover, the water consumption was nearly $1 / 16$ of pit irrigation and 1/24 of drip irrigation, respectively. This technology can significantly reduce water consumption while ensuring the survival rate. Therefore, non-pressure infiltrating irrigation is highly suitable for sand fixing plants in desertification areas with water shortage, and it could play an important role in desertification control.

Author Contributions: Data curation, Y.Q.; formal analysis, Y.Q.; writing—original draft, Y.Q.; writing—review and editing, Y.Q. and Z.Z. All authors have read and agreed to the published version of the manuscript.

Funding: This research was funded by the Chinese National Natural Science Foundation (No. 50772131), the Shanxi Applied Basic Research Project (No. 201801D221147), the Shanxi Science and Technology Major Project (No. 20181101003), Scientific and Technological Innovation Programs of Higher Education Institutions in Shanxi (No. 2019L0567), the Science and Technology Plan Project of Shanxi Provincial Department of Housing and Urban-Rural Development (No. 2018-13) and the Science Research Fund of North University of China.

Acknowledgments: We would like to thank the Institute of Agricultural Sciences in Dengkou for data collection assistance.

Conflicts of Interest: The authors declare no conflict of interest.

\section{References}

1. Reynolds, J.F.; Smith, D.M.S.; Lambin, E.F.; Turner, B.L., II; Mortimore, M.; Batterbury, S.P.J;; Downing, T.E.; Dowlatabadi, H.; Fernández, R.J.; Herrick, J.E.; et al. Global desertification: Building a science for dryland development. Science 2007, 316, 847-851. [CrossRef] [PubMed]

2. D'Odorico, P.; Bhattachan, A.; Davis, K.F.; Ravi, S.; Runyan, C.W. Global desertification: Drivers and feedbacks. Adv. Water Resour. 2013, 51, 326-344. [CrossRef]

3. Zhao, W.; Hu, G.; Zhang, Z.; He, Z. Shielding effect of oasis-protection systems composed of various forms of wind break on sand fixation in an arid region: A case study in the Hexi Corridor, northwest China. Ecol. Eng. 2008, 33, 119-125. [CrossRef]

4. Qiu, G.Y.; Lee, I.B.; Shimizu, H.; Gao, Y.; Ding, G. Principles of sand dune fixation with straw checkerboard technology and its effects on the environment. J. Arid Environ. 2004, 56, 449-464. [CrossRef]

5. Liu, J.; Shi, B.; Lu, Y.; Jiang, H.; Huang, H.; Wang, G.; Kamai, T. Effectiveness of a new organic polymer sand-fixing agent on sand fixation. Environ. Earth Sci. 2012, 65, 589-595. [CrossRef]

6. Lokhande, V.H.; Gor, B.K.; Desai, N.S.; Nikam, T.D.; Suprasanna, P. Sesuvium portulacastrum, a plant for drought, salt stress, sand fixation, food and phytoremediation. A review. Agron. Sustain. Dev. 2013, 33, 329-348. [CrossRef]

7. Han, Y.; Wang, K.; Zhang, R.; Yao, Y.; Fan, C. Study on the transpiration eco-physiological characteristics of degenerated Haloxylon ammodendron in Jilantai. Acta Agrestia Sinica 2002, 10, 40-44.

8. He, X.; Gao, Y.; Duan, Z.; Zhao, A.; Chen, H. The comparison of irrigation manners for constructing sand-fixing plantation along Tarim Desert Highway. Scientia Geographica Sinica 2002, 22, 213-218.

9. Ding, X.; Zhou, Z.; Xu, X.; Wang, Y.; Jiang, Y.; Lu, J.; Chang, Q. Dynamics of soil water and salt in soil under artificial plantation shelterbelt drip-irrigated with saline water in the Center of the Taklimakan Desert. Acta Pedologica Sinica 2016, 53, 103-116.

10. Sun, T.; Han, J.; Zhao, S.; Yue, W. Effect of fertilizer application on seed yield and yield components of Agropyron cristatum. J. China Agric. Univ. 2005, 2005, 15-20.

11. Li, P.; Yang, G.; He, X.; Li, F.; Yan, K.; Wang, Z. Effects of drip irrigation on components of water cycle in arid inland areas: A case study of Manas river basin in northwestern China. Int. J. Agric. Biol. Eng. 2019, 12, 132-138. [CrossRef]

12. Ayars, J.E.; Phene, C.J.; Hutmacher, R.B.; Davis, K.R.; Schoneman, R.A.; Vail, S.S.; Mead, R.M. Subsurface drip irrigation of row crops: A review of 15 years of research at the Water Management Research Laboratory. Agric. Water Manag. 1999, 42, 1-27. [CrossRef]

13. Shen, H.; Li, Z.; Zhang, R.; Li, J.; Zhu, E. The current situation and development trend of ferlilizer-water irrigation technology based on infiltrating irrigation system. Acta Agric. Shanghai 2014, 30, 126-130.

14. Jia, Z.; Lu, Q.; Guo, B.; Zhao, M.; Liang, Y. Progress in the study of psammophyte-Haloxylon. For. Res. 2004, $17,125-132$. 
15. Camp, C.R. Subsurface drip irrigation: A review. Trans. ASAE 1998, 41, 1353-1367. [CrossRef]

16. Capra, A.; Scicolone, B. Emitter and filter tests for wastewater reuse by drip irrigation. Agric. Water Manag. 2004, 68, 135-149. [CrossRef] 